\title{
A Response to the Letter to the Editor Regarding "Nationwide Prevalence of Diabetes and Prediabetes and Associated Risk Factors Among Iranian Adults: Analysis of Data from PERSIAN Cohort Study" to the end of Study
}

\author{
Mohammad E. Khamseh · Sadaf G. Sepanlou • Reza Malekzadeh (D)
}

Received: November 10, 2021 / Accepted: November 23, 2021 / Published online: December 3, 2021

(c) The Author(s) 2021

Keywords: Type 2 diabetes; Prediabetes; Ethnicity; Prevalence

Dear Editor,

We would like to thank the authors of the letter to the editor for their interest in our paper entitled "Nationwide Prevalence of Diabetes and Prediabetes and Associated Risk Factors Among Iranian Adults: Analysis of Data from PERSIAN Cohort Study".

The prevalence of diabetes is increasing worldwide. This has been supported by evidence reported by the International Diabetes Federation (IDF) [1], the National Health and Nutrition Examination Survey (NHANES) [2], and data from the Global Burden of Disease (GBD) study [3]. The prevalence of diabetes in

M. E. Khamseh

Endocrine Research Center, Institute of

Endocrinology and Metabolism, Iran University of

Medical Sciences (IUMS), Tehran, Iran

S. G. Sepanlou

Digestive Disease Research Center, Digestive

Diseases Research Institute, Tehran University of

Medical Sciences, Tehran, Iran

R. Malekzadeh ( $₫)$

Digestive Oncology Research Center, Digestive

Diseases Research Institute, Tehran University of

Medical Sciences, N. Karegar Ave. Shariati Hospital,

14117-13014 Tehran, Iran

e-mail: malek@tums.ac.ir;

dr.reza.malekzadeh@gmail.com
Iranian adults over 25 years of age reported by the GBD study was $4.8 \%$ (95\% UI $4.3-5.3$ ) in 1990 , which increased to over 10\% (9.0-11.1) in 2019. Our study showed that the prevalence of diabetes and prediabetes in Iran is $15.0 \%$ (95\% CI $12.6-17.3)$ and $25.4 \% \quad$ (95\% CI 18.6-32.1), respectively. Additionally, results from recent local studies are in agreement with our results. In a recent study from Tehran, in people 30-75 years of age, the estimated overall prevalence of diabetes was $14.3 \%$ (95\% CI 13.1-15.8). Prediabetes was detected in about $29.2 \%$ of the study participants (95\% CI 22.9-36.3) [4]. In a two-step cluster sampling study in Yazd, of the 9965 individuals recruited aged between 20 and 69 years old, the prevalence of diabetes was $14.1 \%$ (95\% CI 13.4-14.7). Moreover, the prevalence of prediabetes was $25.8 \%$ [5]. In addition, the authors of the letter to the editor confirmed that the reported prevalence of our study is comparable to their result in Tehran [6]. It should be noted that methods and instruments for recording fasting plasma glucose (FPG) were not the same across different iterations of STEPS surveys, while laboratory methods followed unified protocols and instruments for measuring FPG across the PERSIAN cohort centers. In addition, PERSIAN continued till 2020, which explains the reported higher prevalence of prediabetes and diabetes compared with STEPS 2016. 
The authors of the letter to the editor also stated that "Diabetes and prediabetes are different stages in the spectrum of a common disease (dysglycemia)". This is a pathophysiologic approach that might be unmatched with the real-world evidence. Recently, Lord et al. [7] reported geographic disparities of prediabetes and diabetes in Florida. They used multivariable logistic regression models in their study. The prevalence of diabetes was $11.5 \%$ while that of prediabetes was $8.2 \%$. Furthermore, they reported that "although there was an overlap between counties within high-prevalence prediabetes and diabetes clusters, the distribution of the conditions exhibited somewhat differing spatial patterns. The primary prediabetes cluster was large and relatively diverse". Other studies identified geographic and ethnic disparities in the distribution of risk factors for diabetes $[8,9]$. Globally, diabetes prevalence is higher in men than women. However, sex differences in diabetes prevalence vary by geographic area and age group $[1,10]$.

In our study we showed that compared with Fars ethnic background, as the largest ethnic group in our cohort, Balouch and Zaboli individuals were more likely to develop diabetes. On the other hand, Arab and Zaboli individuals were associated with significantly lower odds of prediabetes. Similar discrepancies have been reported in previous reports. In the study by Lord et al. [7], although ethnicity was associated with prediabetes among residents of high-prediabetes clusters, they found no association between ethnicity and diabetes status.

Consanguineous marriage may affect the development of diabetes as one of the genetic factors. Meanwhile, some studies have suggested that observed ethnic disparities may be a reflection of differences in socioeconomic and environmental risk factors that are associated with ethnicity $[11,12]$. It is also worth noting that Iran comprises individuals with different ethnic backgrounds. However, each ethnicity is approximately localized. For example, Zaboli and Balouch individuals live mostly in the southeast of Iran. Arabs reside in the southwest, and Azari Iranians inhabit the northeast of the country, while most with Fars ethnic background reside in central provinces. Therefore, various environmental and lifestyle risk factors are associated with each ethnicity. In addition to genetic characteristics, there may be several other confounders such as cultural, behavioral, and environmental risk factors for which we cannot adjust. Family history is one such factor. Therefore, we reported odds ratios for ethnicities with the understanding that many other confounders may exist and may be associated with each specific ethnicity. These include but are not limited to consanguineous marriage, accessibility of healthcare services, geographic characteristics, and dietary intake pattern. Considering the cross-sectional nature of our study, our results do not imply a causal inference.

Regarding the statistical methods, we compared people with prediabetes and diabetes with those who had normoglycemia using real-world data. It is correct that ordinal and multinomial logistic models could be used. However, binary logistic regression models for prediabetes versus normal glycemic participants and diabetes versus non-diabetes are statistically robust. Furthermore, we included hypertension in the logistic regression models. Hypertension was associated neither with diabetes nor with prediabetes and did not improve the performance of the model. We did not adjust the model for cardiovascular diseases (CVDs) because CVDs are generally considered to be a consequence of prediabetes and diabetes that occur later in the causal graph and hence are not determinants for prediabetes and diabetes [13, 14]. The authors of the letter to the editor have cited their own study [6] which examines the prevalence of coronary heart disease across different levels of glycemic status. This study confirms that the authors have also considered CVD as the consequence of dysglycemia and not as a determinant.

Current evidence shows that central obesity has a different impact on cardiometabolic disorders than body mass index (BMI). Central obesity is associated with stronger inflammation than general obesity, which might make a greater contribution to the risk of related disorders [15-17]. In our analyses, we simultaneously included both the BMI and measures of 
central obesity in the models. We ruled out their collinearity in our models as well.

Baseline characteristics of the study participants were given in Table 1 of the supplementary material of our paper. PERSIAN is a large population-based study in various areas of Iran with diverse ethnicities, cultures, and environmental risk factors. Our study was conducted in a large population-based sample in Iran following a robust quality assurance and control program.

In conclusion, despite what Asgari et al. claimed, our results and the literature in the field confirm an increasing trend in prevalence of diabetes and prediabetes in Iran. Moreover, similar discrepancies have been reported previously in relation to the observed ethnic disparities. To the best of our knowledge, this is the first study that included major ethnic groups in Iran and provides a direct comparison of diabetes/prediabetes prevalence among different ethnicities. We hope that all questions and points in the letter have been addressed.

\section{ACKNOWLEDGEMENTS}

Funding. No funding or sponsorship was received for writing or publication of this letter.

Authorship. All named authors meet the International Committee of Medical Journal Editors (ICMJE) criteria for authorship for this article, take responsibility for the integrity of the work as a whole, and have given their approval for this version to be published.

Authors' Contributions. Mohammad E. Khamseh and Sadaf G. Sepanlou designed and wrote the draft, Reza Malekzadeh critically revised the letter.

Disclosures. Mohammad E. Khamseh, Sadaf G. Sepanlou, and Reza Malekzadeh have nothing to disclose.

Compliance with Ethics Guidelines. This article is a letter regarding our previous paper entitled "Nationwide Prevalence of Diabetes and Prediabetes and Associated Risk Factors Among Iranian Adults: Analysis of Data from PERSIAN Cohort Study". PERSIAN was approved by the ethics committees of the Digestive Disease Research Institute in Tehran University of Medical Sciences and the Medical Sciences Universities supervising each cohort in local study centers. The study was performed in accordance with the Helsinki Declaration and its later amendments. Informed consent was obtained from all individual participants included in the study. This particular article is based on previously conducted studies and does not contain any new studies with human participants or animals performed by any of the authors.

Data Availability. Data sharing is not applicable to this article as no datasets were generated or analyzed to write this letter.

Open Access. This article is licensed under a Creative Commons Attribution-NonCommercial 4.0 International License, which permits any non-commercial use, sharing, adaptation, distribution and reproduction in any medium or format, as long as you give appropriate credit to the original author(s) and the source, provide a link to the Creative Commons licence, and indicate if changes were made. The images or other third party material in this article are included in the article's Creative Commons licence, unless indicated otherwise in a credit line to the material. If material is not included in the article's Creative Commons licence and your intended use is not permitted by statutory regulation or exceeds the permitted use, you will need to obtain permission directly from the copyright holder. To view a copy of this licence, visit http://creativecommons.org/licenses/by$\mathrm{nc} / 4.0 /$.

\section{REFERENCES}

1. Saeedi P, Petersohn I, Salpea P, et al. Global and regional diabetes prevalence estimates for 2019 and projections for 2030 and 2045: results from the International Diabetes Federation Diabetes Atlas. Diabetes Res Clin Pract. 2019;157:107843. 
2. Zhang N, Yang X, Zhu X, Zhao B, Huang T, Ji Q. Type 2 diabetes mellitus unawareness, prevalence, trends and risk factors: National Health and Nutrition Examination Survey (NHANES) 1999-2010. J Int Med Res. 2017;45(2):594-609.

3. GBD 2019 Diseases and Injuries Collaborators. Global burden of 369 diseases and injuries in 204 countries and territories, 1990-2019: a systematic analysis for the Global Burden of Disease Study 2019. Lancet. 2020;396(10258):1204-1222.

4. Barati S, Sadeghipour P, Ghaemmaghami Z, et al. Warning signals of elevated prediabetes prevalence in the modern Iranian urban population. Prim Care Diabetes. 2021;15(3):472-9.

5. Mirzaei M, Rahmaninan M, Mirzaein M, Nadjarzadeh A, Dehghani-Tafti AA. Epidemiology of diabetes mellitus, pre-diabetes, undiagnosed and uncontrolled diabetes in Central Iran: results from Yazd health study. BMC Public Health. 2020;20: 166.

6. Moazzeni SS, Ghafelehbashi H, Hasheminia M, et al. Sex-specific prevalence of coronary heart disease among Tehranian adult population across different glycemic status: Tehran lipid and glucose study, 2008-2011. BMC Public Health. 2020;20(1): 1510 .

7. Lord J, Roberson S, Odoi A. Investigation of geographic disparities of pre-diabetes and diabetes in Florida. BMC Public Health. 2020;20(1):1226.

8. Ford ES, Mokdad AH, Giles WH, Galuska DA, Serdula MK. Geographic variation in the prevalence of obesity, diabetes, and obesity-related behaviors. Obes Res. 2005;13(1):118-22.

9. Voeks JH, McClure LA, Go RC, et al. Regional differences in diabetes as a possible contributor to the geographic disparity in stroke mortality: the REasons for Geographic And Racial Differences in Stroke Study. Stroke. 2008;39(6):1675-80.
10. Centers for Disease Control and Prevention. National Diabetes Statistics Report 2020. Atlanta, GA: Centers for Disease Control and Prevention, U. S. Department of Health and Human Services, 2020. https://www.cdc.gov/diabetes/library/ features/diabetes-stat-report.html.

11. Link CL, McKinlay JB. Disparities in the prevalence of diabetes: Is it race/ethnicity or socioeconomic status? Results from the Boston Area community health (BACH) survey. Ethn Dis. 2009;19(3): 288-92.

12. Signorello LB, Schlundt DG, Cohen SS, et al. Comparing diabetes prevalence between African Americans and Whites of similar socioeconomic status. Am J Public Health. 2007;97(12):2260-7.

13. Centers for Disease Control and Prevention. National diabetes fact sheet: national estimates and general information on diabetes and prediabetes in the United States, 2011. Atlanta, GA: Department of Health and Human Services, Centers for Disease Control and Prevention, 2011.

14. Wannamethee SG, Shaper AG, Whincup PH, Lennon L, Sattar N. Impact of diabetes on cardiovascular disease risk and all-cause mortality in older men: influence of age at onset, diabetes duration, and established and novel risk factors. Arch Intern Med. 2011;171(5):404-10.

15. Wang Y, Rimm EB, Stampfer MJ, Willett WC, $\mathrm{Hu}$ FB. Comparison of abdominal adiposity and overall obesity in predicting risk of type 2 diabetes among men. Am J Clin Nutr. 2005;81(3):555-63.

16. Liu D, Li Q, Dong J, et al. The association between normal BMI with central adiposity and proinflammatory potential immunoglobulin G N-glycosylation. Diabetes Metab Syndr Obes. 2019;12:2373-85.

17. Mamtani MR, Kulkarni HR. Predictive performance of anthropometric indexes of central obesity for the risk of type 2 diabetes. Arch Med Res. 2005;36(5): 581-9. 